\title{
Reliability and Accuracy of Palpable Anterior Neck Landmarks for the Identification of Cervical Spinal Levels
}

\author{
Koopong Siribumrungwong, Chitpon Sinchai, Boonsin Tangtrakulwanich, Weera Chaiyamongkol \\ Department of Orthopaedic and Physical Medicine, Faculty of Medicine, Prince of Songkla University, Hadyai, Thailand
}

\section{Study Design: A descriptive experimental study.}

Purpose: The purpose of this study was to describe the reliability and accuracy of palpable anterior neck landmarks (angle of the mandible, hyoid bone, thyroid cartilage, and cricoid cartilage) for the identification of cervical spinal levels in a slight neck-extended position as in anterior approach cervical spinal surgery.

Overview of Literature: Standard, palpable anatomical landmarks for the identification of cervical spinal levels were described by Hoppenfeld using the midline palpable anterior structures (angle of the mandible [C2 body], hyoid bone [C3 body], thyroid cartilage [C4C5 disc], cricoid cartilage [C6 body], and carotid tubercle [C6 body]) to determine the approximate level for skin incisions. However, in clinical practice, patients are positioned with a slight neck extension to achieve cervical lordosis. This positioning (neck extension) may result in changes in the locations of anatomical landmarks compared with those reported in previous studies.

Methods: This experimental study was conducted on 96 volunteers. Each volunteer was palpated for locating four anatomical landmarks three times by three different orthopedic surgeons. We collected data from the level of the vertebral body or the vertebral disc matching the surface anatomical landmarks from the vertical reference line.

Results: Accuracy of the angle of the mandible located at the C2 vertebral body was $95.5 \%$, the hyoid bone located at the C2/3 intervertebral disc was $51.7 \%$, the thyroid cartilage located at the C4 vertebral body was $42 \%$, and the cricoid cartilage located at the C5/6 intervertebral disc was $43.4 \%$.

Conclusions: With the neck in a slightly extended position to achieve cervical lordosis, the angle of the mandible, the hyoid bone, the thyroid cartilage, and the cricoid cartilage were most often located at the $\mathrm{C} 2$ body, the C2/3 disc, the C4 body, and the C5/6 disc, respectively. The angle of the mandible and the hyoid bone are highly reliable surface anatomical landmarks for the identification of cervical spinal levels than the thyroid cartilage and the cricoid cartilage.

Keywords: Cervical spine; Anatomic landmarks

\section{Introduction}

Anterior approach cervical spine surgeries are commonly performed by both neurosurgeons and orthopedic surgeons in procedures such as anterior cervical discectomies and anterior cervical corpectomies. Smith and Robinson

Received Apr 27, 2017; Revised Jun 8, 2017; Accepted Jun 17, 2017

Corresponding author: Koopong Siribumrungwong

Department of Orthopaedic and Physical Medicine, Faculty of Medicine, Prince of Songkla University, Hadyai, Songkhla 90110, Thailand

Tel: +66-902016565, Fax: +66-29269793, E-mail: koopongs@hotmail.com 
[1] reported the anterior approach to the cervical spine using a transverse skin incision in 1955. The advantages of an anterior approach include direct removal of the compression lesion with minimal muscle dissection, low rates of wound complications, and postoperative pain [2]. A transverse skin incision is preferred for cosmetic reasons and generally used for one- or two-level lesions. However, one of its main disadvantages is that it is a relatively less extensile approach, making the exact identification of the skin incision site a key factor to minimize soft tissue injury. Moreover, it can be performed more easily and has a shorter operative time compared with other procedures. Skin incisions done at an inaccurate level may lead to increased technical difficulties during the surgical procedure, increased risks of esophageal injury due to excessive retraction, inadequate spinal decompression, and dysphagia [3].

Standard, palpable anatomical landmarks for the identification of cervical spinal levels were described by Hoppenfeld and DeBoer [4] using the midline, palpable anterior structures. These structures are the angle of the mandible ( $\mathrm{C} 2$ body), the hyoid bone ( $\mathrm{C} 3$ body), the thyroid cartilage ( $\mathrm{C} 4-\mathrm{C} 5 \mathrm{disc})$, the cricoid cartilage (C6 body), and the carotid tubercle (C6 body), and these are used to determine the approximate level for skin incisions. Several studies have reported that the carotid tubercle can be used as an anatomical landmark for the sixth cervical vertebra [5], but it is deeply located and is difficult to palpate [6]. However, in clinical practice, patients are positioned with a slight neck extension to achieve cervical lordosis. This positioning (neck extension) may result in changes in the locations of anatomical landmarks compared with the results described earlier by Hoppenfeld and DeBoer [4]. To the best of our knowledge, only a few studies have reported on palpable, anatomical landmarks in the neck for the identification of cervical spinal levels [5-7]. However, in our review of the literature, we were unable to find any rigorous studies specifically evaluating the surface anatomical landmarks as they relate to vertebral levels in a true surgical position.

The purpose of this study was to describe the reliability and accuracy of the palpable, anterior neck landmarks (angle of the mandible, hyoid bone, thyroid cartilage, and cricoid cartilage) for the identification of cervical spinal levels. Our study was performed in a slightly extended neck position similar to that during anterior approach cervical spinal surgery.

\section{Materials and Methods}

This is a descriptive experimental study comprising a population of 96 volunteers recruited in the period from March 2012 to December 2013. The inclusion criterion was healthy volunteers aged between 30 and 80 years. The exclusion criteria were revision cervical spine surgery, the presence of congenital anomalies (i.e., Klippel-Feil syndrome), pregnancy, or cervical trauma. This study was approved by the Ethics Committee of the Faculty of Medicine, Prince of Songkla University (IRB no., EC 56-03511-1-2). A written, informed consent form was obtained from all volunteers.

The primary outcome of this study was reliability of anatomical landmarks in identifying the cervical spinal levels. The secondary outcome was its accuracy. The volunteers' demographic data included age, gender, weight, height, and body mass index (BMI). All volunteers were placed in a supine position on an actual operative table. Two towel rolls were placed under the posterior aspect of the neck and adjusted to variable heights until the desired cervical lordosis was achieved. The neck was positioned in 20-30 degrees of extension measured by goniometry to achieve normal cervical lordosis without rotation of the head (Fig. 1). Each volunteer was palpated for locating four anatomical landmarks (angle of the mandible, hyoid bone, thyroid cartilage, and cricoid cartilage) on three occasions by three different orthopedic surgeons. The angle of the mandible reference point was defined as the confluence of the mandibular ramus and body. The hyoid bone,

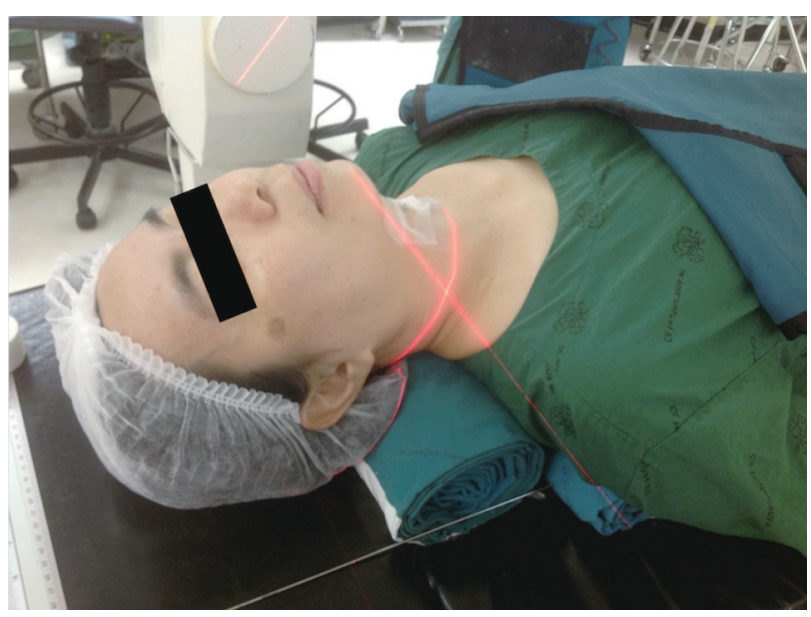

Fig. 1. Volunteers were positioned in a supine position on an operating table with their necks in a slightly extended position to achieve normal cervical lordosis. 
thyroid cartilage, and cricoid cartilage reference points were defined as the most prominent point of the hyoid bone, thyroid cartilage, and cricoid cartilage, respectively, in the midline of the patient. Each orthopedic surgeon marked the four anatomical landmarks with radiopaque markers, and a lateral cervical radiograph was obtained using intraoperative $\mathrm{C}$-arm fluoroscopy.

Radiographic measurements were analyzed by a single observer. Vertical lines were drawn on each of the radiographs from the four markers to the cervical vertebral body or disc space (Fig. 2). We collected data from each level of the vertebral body or the vertebral disc matching the surface anatomical landmarks from the referenced vertical line.

The software SPSS ver. 9.0 (SPSS Inc., Chicago, IL, USA) was used for statistical analysis. The kappa statistical method was applied to compare results obtained by the three orthopedic surgeons, and a $p$-value of $<0.05$ was considered statistically significant.

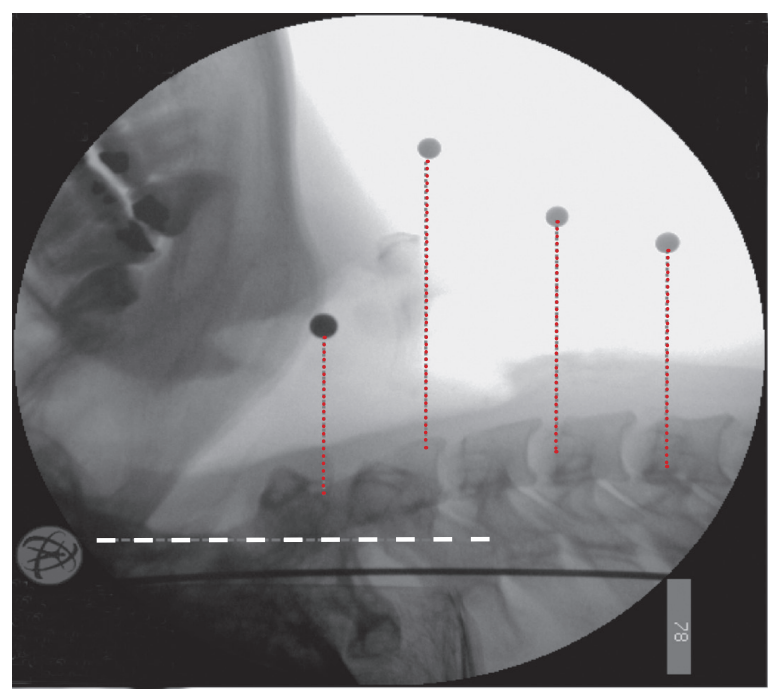

Fig. 2. Each of the radiographs had vertical lines drawn from four markers to the cervical vertebral body or disc space.

\section{Results}

\section{Baseline characteristics}

A total of 96 volunteers participated in this study. Their mean age was $43.2 \pm 9.8$ years, and the mean BMI was $23.5 \pm 3.7 \mathrm{~kg} / \mathrm{m}^{2}$. The demographic data of the volunteers are shown in Table 1.

\section{Interobserver agreement}

This study used the kappa coefficient to evaluate interobserver agreement among the three orthopedic surgeons regarding the four anatomical landmarks (Table 2). Statistical analysis revealed an overall kappa value of 0.679 and 0.753 with $p<0.001$ for the angle of the mandible and hyoid bone, respectively. This indicates that these two anatomical landmarks show substantial agreement [8]. Statistical analysis revealed an overall kappa value of 0.557 and 0.542 with $p<0.001$ for the thyroid cartilage and the cricoid cartilage, respectively. This indicated moderate agreement between these two anatomical landmarks [8].

\section{Accuracy of the four anatomical landmarks}

The angle of the mandible located at the $\mathrm{C} 2$ vertebral body had an accuracy of $95.5 \%$ (275/288). The hyoid bone,

Table 1. Demographic data ( $\mathrm{N}=96)$

\begin{tabular}{lc} 
Characteristic & Value \\
\hline Age $(\mathrm{yr})$ & $43.27 \pm 9.86$ \\
Gender & \\
\hline Male & $47(49)$ \\
Female & $49(51)$ \\
Weight $(\mathrm{kg})$ & $63.29 \pm 12.57$ \\
Height $(\mathrm{m})$ & $1.63 \pm 0.08$ \\
\hline Body mass index $\left(\mathrm{kg} / \mathrm{m}^{2}\right)$ & $23.55 \pm 3.69$ \\
\hline
\end{tabular}

Values are presented as mean \pm standard deviation or number (\%).

Table 2. Interobserver agreement regarding the four anatomical landmarks

\begin{tabular}{|c|c|c|c|}
\hline Landmarks & Overall kappa & Standard error & $p$-value \\
\hline Angle of the mandible & 0.679 & 0.056 & $<0.001$ \\
\hline Hyoid bone & 0.753 & 0.042 & $<0.001$ \\
\hline Thyroid cartilage & 0.557 & 0.036 & $<0.001$ \\
\hline Cricoid cartilage & 0.542 & 0.038 & $<0.001$ \\
\hline
\end{tabular}


Table 3. Accuracy of cases where each anatomical landmark was located at specific vertebral levels

\begin{tabular}{|c|c|c|c|c|}
\hline Vertebral level & Angle of the mandible & Hyoid bone & Thyroid cartilage & Cricoid cartilage \\
\hline C2 body & $275(95.5)$ & $83(28.8)$ & - & - \\
\hline $\mathrm{C} 2 / 3$ disc & $12(4.2)$ & $149(51.7)$ & - & - \\
\hline C3 body & $1(0.3)$ & $50(17.4)$ & $24(8.3)$ & - \\
\hline C3/4 disc & - & $6(2.1)$ & $90(31.2)$ & - \\
\hline C4 body & - & - & $121(42.0)$ & - \\
\hline C4/5 disc & - & - & $39(13.5)$ & $20(6.9)$ \\
\hline C5 body & - & - & $14(4.9)$ & 95 (32.9) \\
\hline C5/6 disc & - & - & - & $125(43.4)$ \\
\hline C6 body & - & - & - & 44 (15.3) \\
\hline C6/7 disc & - & - & - & $4(1.4)$ \\
\hline C7 body & - & - & - & - \\
\hline
\end{tabular}

Values are presented as number (\%).

primarily located at the $\mathrm{C} 2 / 3$ intervertebral disc, had an accuracy of $51.7 \%$ (149/288). The thyroid cartilage landmark located at the $\mathrm{C} 4$ vertebral body had an accuracy of $42 \%(121 / 188)$. The cricoid cartilage located at the C5/6 intervertebral disc had an accuracy of 43.4\% (125/288) (Table 3).

\section{Discussion}

Anterior approach cervical spine surgery is a common procedure. Clinical success rate of these operations is high and they have a low incidence of complications [9]. The anterior cervical spine approach has several modifications, and the Smith-Robinson approach is the most common [1]. Proper placement of the incision can maximize exposure of the surgical field and reduce risks of developing pressure-related complications in the neck.

Hoppenfeld and DeBoer [4] described the surface anatomical landmarks (hyoid bone [C3], thyroid cartilage [C4-C5], cricoid cartilage [C6] and carotid tubercle [C6]). Auerbach et al. [6] described the angle of the mandible as the anatomical landmark for the C2/3 disc level. Our study showed slightly higher locations of these landmarks compared with previous studies, with the angle of the mandible at the $\mathrm{C} 2$ body, the hyoid bone at the $\mathrm{C} 2 / 3$ disc, the thyroid cartilage at the $\mathrm{C} 4$ body, and the cricoid cartilage at the $\mathrm{C} 5 / 6$ disc. These differences may be due to the placement of the volunteers in a position with a slight neck extension (positioned to achieve cervical lordosis), an upward motion of the mobile structures including the hyoid bone, thyroid cartilage, and cricoid cartilage occurred.
We did not include the carotid tubercle as an anatomical landmark in this study because several studies have reported that in obese or muscular patients, or those with short and wide necks, surgeons might find this landmark not easily palpated $[6,10]$. However, an advantage of using the carotid tubercle as an anatomical landmark is that it is not affected by the positioning of the cervical spine during surgery.

Our study has shown that the angle of the mandible and the hyoid bone are highly reliable (substantial interobserver agreement) surface anatomical landmarks than the cricoid and the thyroid cartilage (moderate interobserver agreement). The angle of the mandible is a fixed bony landmark if there is no mastication, speech, or swallowing [11]. The hyoid bone is only distantly articulated to other bones by muscles or ligaments. The hyoid is anchored by nine muscles from the anterior, posterior, and inferior directions and connected to the styloid processes of the temporal bone through stylohyoid ligaments [12]. In comparison, the thyroid and cricoid cartilages are more mobile and no strong ligaments are connected to fixed bony anatomy, which could reasonably account for these two anatomical landmarks having less reliability.

However, we acknowledge that we used an intraoperative $\mathrm{C}$-arm fluoroscope with a radiopaque marker to accurately identify the operative level before making the incision. We consider surface anatomical landmarks to be useful in guiding the location of radiopaque markers and reducing the radiation dose and overall surgical time.

One limitation of this study was the inability to include the $\mathrm{C} 7 / \mathrm{T} 1$ disc level in the analysis due to poor-quality 
results obtained using intraoperative $\mathrm{C}$-arm fluoroscopy. Moreover, in our study, no surface anatomical landmark was located below the C7 vertebral body. Another limitation of this study was the fact that it did not include obese patients $\left(\mathrm{BMI}>30 \mathrm{~kg} / \mathrm{m}^{2}\right)$ or those with short necks.

\section{Conclusions}

When the neck was in a slightly extended position to achieve cervical lordosis, the angle of the mandible, the hyoid bone, the thyroid cartilage, and the cricoid cartilage were primarily located at the $\mathrm{C} 2$ body, C2/3 disc, C4 body, and $\mathrm{C} 5 / 6$ disc, respectively. The angle of the mandible and the hyoid bone are highly reliable surface anatomical landmarks for the identification of cervical spinal levels than the thyroid cartilage and the cricoid cartilage.

\section{Conflict of Interest}

No potential conflict of interest relevant to this article was reported.

\section{References}

1. Smith GW, Robinson RA. The treatment of certain cervical-spine disorders by anterior removal of the intervertebral disc and interbody fusion. J Bone Joint Surg Am 1958;40-A:607-24.

2. Rhee JM, Yoon T, Riew KD. Cervical radiculopathy. J Am Acad Orthop Surg 2007;15:486-94.

3. Tortolani PJ, Cunningham BW, Vigna F, Hu N, Zorn $\mathrm{CM}$, McAfee PC. A comparison of retraction pressure during anterior cervical plate surgery and cervical disc replacement: a cadaveric study. J Spinal Disord Tech 2006;19:312-7.
4. Hoppenfeld S, DeBoer P. Surgical exposures in orthopaedics: the anatomic approach. 3rd ed. Philadelphia: Lippincott Williams \& Wilkins; 2003.

5. Lee JH, Lee JH, Lee HS, Lee DY, Lee DO. The efficacy of carotid tubercle as an anatomical landmark for identification of cervical spinal level in the anterior cervical surgery: comparison with preoperative Carm fluoroscopy. Clin Orthop Surg 2013;5:129-33.

6. Auerbach JD, Weidner Z, Pill SG, Mehta S, Chin KR. The mandibular angle as a landmark for identification of cervical spinal level. Spine (Phila Pa 1976) 2009;34:1006-11.

7. Leonard M, Kennedy C, Heneghan H, Cabe JM. The application of a radiographically determined ratio as a new technique to identify the optimal level of transverse skin incision for anterior cervical spine surgery. J Clin Neurosci 2012;19:1278-80.

8. Landis JR, Koch GG. The measurement of observer agreement for categorical data. Biometrics 1977;33:159-74.

9. Daniels AH, Riew KD, Yoo JU, et al. Adverse events associated with anterior cervical spine surgery. J Am Acad Orthop Surg 2008;16:729-38.

10. Cha YD, Lee SK, Kim TJ, Han TH. The neck crease as a landmark of Chassaignac's tubercle in stellate ganglion block: anatomical and radiological evaluation. Acta Anaesthesiol Scand 2002;46:100-2.

11. Chin KR, Auerbach JD, Adams SB Jr, Sodl JF, Riew KD. Mastication causing segmental spinal motion in common cervical orthoses. Spine (Phila Pa 1976) 2006;31:430-4.

12. Andrade AV, Gomes PF, Teixeira-Salmela LF. Cervical spine alignment and hyoid bone positioning with temporomandibular disorders. J Oral Rehabil 2007;34:767-72. 